\title{
Time to Wealth Goals in Capital Accumulation*
}

\author{
Leonard C. MacLean \\ Imaclean@mgmt.dal.ca \\ School of Business Administration \\ Dalhousie University \\ William T. Ziemba \\ ziemba@interchange.ubc.ca \\ Faculty of Commerce and Business Administration \\ University of British Columbia \\ Yuming Li \\ School of Business \\ California State University at Fullerton
}

\begin{abstract}
$\underline{\text { ABSTRACT }}$
This paper considers the problem of continuous investment of capital in risky assets over time. Using a Bayesian framework, a model for asset prices is developed where the current price dynamics depend on the history of realized prices. A dynamic Bayesian fractional Kelly strategy, where the investor rebalances the portfolio based on the performance to date, is shown to be optimal assuming that the risky assets are jointly lognormally distributed. The strategy minimizes the expected time to reach a wealth goal while maintaining a high probability of reaching that goal before falling to a subsistence level of wealth.

*This research was supported by the National Sciences and Engineering Research Council of Canada. Thanks go to Yonggan Zhao for comments on an earlier draft of the paper.
\end{abstract}




\section{1- INTRODUCTION}

In capital accumulation under uncertainty, a decision-maker must determine how much capital to invest in riskless and risky investment opportunities at each point in time. The investment strategy yields a stream of capital over a planning horizon, with investment decisions made so that the distribution of wealth over time has desirable properties. An investment strategy which has generated considerable interest is the growth optimal or Kelly strategy, where the expected logarithm of wealth is maximized in each period. The wealth distribution of this strategy has many attractive characteristics (see, e.g. Hakansson, 1970, 1971; and Markowitz, 1976). As Breiman (1960, 1961), and Algeot and Cover (1988) have shown, the Kelly (1956) strategy maximizes the long run expected rate of growth of capital and minimizes the expected time to reach a fixed level of wealth for sufficiently large goals under mild conditions. Researchers such as Thorp (1975), Hausch, Ziemba and Rubinstein (1981), Grauer and Hakansson (1986, 1987), and Mulvey and Vladimirou (1992) have used the optimal growth strategy to compute optimal portfolio weights in multi-asset and worldwide asset allocation problems.

The stream of capital following from an investment policy can be viewed from either a wealth or time perspective. Thus the distribution of accumulated capital to a fixed point in time and the distribution of first passage time to a fixed level of accumulated capital are variables controlled by the investment decisions. 
The Kelly strategy controls (optimizes) the expected values of those distributions. However, the expected log strategy is very aggressive; this is seen because the Arrow-Pratt risk aversion index for the logarithm is the reciprocal of wealth which is essentially zero for typical wealth levels. As Hausch and Ziemba (1985) and Clark and Ziemba (1987) have demonstrated, the optimal portfolio weights in the risky assets given by this strategy tend to be so large for favorable investments that the chances of losing a substantial portion of wealth are very high, particularly if the probability estimates are in error.

A standard approach to controlling risk is to include the variance of the wealth or time distributions in the analysis. Mean-variance analysis of wealth has been widely used to determine investment strategies; see Markowitz, $1952,1987$. In the time domain the mean-variance approach yields somewhat different strategies. However, the logarithm of wealth and first passage time have consistent mean-variance properties; see Burkhardt, 1998.

An alternative to characterizing distributions with mean and variance is to use quantiles. MacLean, Ziemba, and Blazenko (1992) considered quantiles for wealth, $\log$ wealth and first passage time in identifying investment strategies which achieve capital growth with a required level of security. Growth can be traded for security with fractional Kelly strategies. In discrete time models with general 
return distributions this strategy is suboptimal but has attractive wealth/time distribution properties.

A limitation of this research is that the probability distributions for risky asset returns are fixed. In a more general setting the parameters of the return distribution are random. Browne and Whitt (1997) considered a random parameter model for a single risky asset. This flexible framework accommodates realistic asset prices and provides a mechanism for the dynamic learning of an asset price distribution; see Brennan, 1998.

In this paper the passage time to specified wealth levels is analyzed in a dynamic stochastic model for wealth accumulation. The model is a generalization to the multi-asset case of the random coefficients model. With reference to the distribution of first passage time, a fractional Kelly investment strategy is developed which minimizes the expected time to a target level of wealth while maintaining a high probability of achieving the goal before falling to a subsistence level of wealth. The strategy is a blend of the risk free (cash) strategy and the optimal growth strategy based on the most current information on asset prices. This optimality of the fractional Kelly strategy is dependent upon the lognormality of the prices for risky assets and is not in general valid without this assumption. 


\section{2 - BAYESIAN ASSET PRICING MODEL}

Consider an investor who allocates current wealth to various assets in a competitive capital market, where the trading of assets takes place continuously in time. If the trading price of asset $i$ at time $\mathrm{t}$ is $\mathrm{P}_{\mathrm{i}}(\mathrm{t})$, then consider the logarithm of prices $Y_{i}(t)=\ln P_{i}(t), i=1, \ldots, p$. Assume that the $Y_{i}(t)$ follow the stochastic differential equations

$$
d Y_{i}=\alpha_{i} d t+\delta_{i} d Z_{i}, \quad i=1, \ldots, p
$$

where $\mathrm{Z}_{\mathrm{i}}$ is standard Brownian motion.

With $Y^{\prime}(t)=\left(Y_{1}(t), \ldots, Y_{p}(t)\right), \alpha^{\prime}=\left(\alpha_{1}, \ldots, \alpha_{p}\right)$ and $\Delta=\operatorname{diag}\left(\delta_{1}^{2}, \ldots, \delta_{p}^{2}\right)$, the conditional distribution of $\log$ returns at time t, given $\alpha$ and $\Delta$, is

$$
Y(t) \mid \alpha, \Delta \sim N(\alpha t, t \Delta)
$$

The mean $\alpha$ is assumed to be a random vector with prior distribution

$$
\alpha \sim N(\mu, \Gamma)
$$

where $\mu^{\prime}=\left(\mu_{1}, \ldots, \mu_{p}\right)$ and $\Gamma=\left(\gamma_{i j}\right)$, with $\gamma_{i j}$ the covariance between $\alpha_{i}$ and $\alpha_{j}$. For the unconditional distribution of $\log$ returns the covariance is $\Sigma_{t}=t \Delta+t^{2} \Gamma$. In this model the correlation between assets is generated by the random means.

Based on the prior distribution (3) for $\alpha$ and the conditional distribution (2) for $\mathrm{Y}$, the posterior distribution for $\alpha$ given the data $\mathrm{Y}(\mathrm{t})$ is

$$
\alpha \mid Y \sim N\left(\mu_{t}, \Gamma_{t}\right), \text { where }
$$




$$
\begin{aligned}
& \mu_{t}=\mu+\left(I-\Delta_{t} \Sigma_{t}^{-1}\right)\left(\bar{Y}_{t}-\mu\right) \\
& \Gamma_{t}=\frac{1}{t^{2}}\left(I-\Delta_{t} \Sigma_{t}^{-1}\right) \Delta_{t}, \\
& \bar{Y}_{t}=\frac{1}{t} Y(t), \Delta_{t}=t \Delta, \text { and } \Sigma_{t}=t \Delta+t^{2} \Gamma .
\end{aligned}
$$

The asset price structure has the following properties.

1. Consider $\Sigma_{t}=t \Delta+t^{2} \Gamma$, the covariance matrix for the logarithm of return on assets. If $\operatorname{rank}(\Gamma)=\mathrm{m}<\mathrm{p}$, then there exist $\mathrm{m}$ funds with $\log$ returns $\mathrm{F}^{\prime}=$ $\left(F_{1}, \ldots, F_{m}\right)$ so that $Y=\mu+\Lambda F+\xi$, where $\Lambda$ is a pxm matrix, $t^{2} \Gamma=\Lambda \Lambda^{\prime}$, and covariance $\xi=\mathrm{t} \Delta$. If $\mathrm{m}$ is small then the structure of asset prices is explained by a few mutual funds with returns composed of combinations of the asset returns. The simplest case has $\mathrm{m}=1$ and the asset prices are determined by a market portfolio.

2. If $\mathfrak{I}_{t}^{Y}=\sigma\left\{Y_{s}, 0 \leq s \leq t\right\}$ is the filtration from (2), then $\mathrm{Y}(\mathrm{t})$ can be represented as

$$
d Y=\mu_{t} d t+\Delta^{1 / 2} d Z
$$

for $d Y^{\prime}=\left(d Y_{1}, \ldots, d Y_{p}\right), \mu_{t}^{\prime}=\left(\mu_{1 t}, \ldots, \mu_{p t}\right)$ and $d \hat{Z}^{\prime}=\left(d \hat{Z}_{1}^{\prime}, \ldots, d \hat{Z}_{p}\right)$; see Kallianpur, 1980. The significance of the new representation for the dynamics of asset prices lies in the fact that the expected rate of return at time $t$ is given by the posterior mean $\mu_{\mathrm{t}}$. As the price process is observed over time, the posterior 
mean is revised. This has been interpreted in the single risky asset case as a learning process (Brennan, 1998), with investment decisions at points in time depending upon knowledge.

3. The drift in the stochastic differential equation (7) has the form $\mu_{t}=\mu+\Phi_{t}\left(\bar{Y}_{t}-\mu\right)$, so the price dynamics exhibit a "reversion" to the longterm mean $\mu$. This is an observed aspect of price behavior, particularly for commodities.

4. The Bayesian framework for asset prices and the posterior mean in (5) lead naturally to empirical Bayes estimators for the rate of return; see Efron and Morris, 1972 and MacLean and Weldon, 1996. For example, assuming a common prior mean, the Stein estimator is

$$
\hat{\mu}_{t}=\overline{\bar{Y}}_{t}+\left(I-k S_{t}^{-1}\right)\left(\bar{Y}_{t}-\overline{\bar{Y}}_{t}\right)
$$

where $\overline{\bar{Y}}_{t}$ is the overall mean and $\mathrm{S}_{\mathrm{t}}$ is the covariance matrix for log returns. Grauer and Hakansson (1986) demonstrated the superior returns of the growth optimal strategy with Stein estimates for the asset prices.

When there exist $\mathrm{m}$ independent mutual funds with returns $\mathrm{F}^{\prime}=\left(\mathrm{F}_{1}, \ldots, \mathrm{F}_{\mathrm{m}}\right)$ so that $\mathrm{Y}=\mu+\Lambda \mathrm{F}+\varepsilon$, then the covariance matrix can be factored. The factor analysis of $\mathrm{S}_{\mathrm{t}}$ yields estimates $\mathrm{L}$ for $\Lambda$ and $\mathrm{D}$ for $\mathrm{t} \Delta$. With $\mathrm{S}^{*}=\mathrm{LL}^{\prime}+\mathrm{D}$, an alternative empirical Bayes estimator is 


$$
\hat{\hat{\mu}}_{t}=\overline{\bar{Y}}_{t}+\left(I-D S^{*-1}\right)\left(\bar{Y}_{t}-\overline{\bar{Y}}_{t}\right) .
$$

This latter estimator uses the correlation between assets to improve the estimates from maximum likelihood and Stein; see MacLean and Weldon, 1996.

\section{3 - CAPITAL ACCUMULATION}

Capital is accumulated through asset investment. It is assumed that the buying and selling of assets takes place continuously in time with no transactions costs or taxes, assets are infinitely divisible, have limited liability, short selling is permitted, and borrowing and lending are allowed at the same rate.

In addition to the opportunity to invest in the risky assets, an individual can allocate capital to a risk free asset (cash) which has a known rate of return $r$, the same as the rate on borrowing and lending.

The focus of the analysis of capital accumulation will be the current investment decision. Given the history of asset prices and accumulated capital from past decisions, the investor allocates capital to the various investment opportunities. The price dynamics given current information are characterized by the conditional (posterior) means as defined in (5). With current level of wealth $\mathrm{W}_{\mathrm{t}}$ and the proportion of wealth invested in assets 1 to $p+1$ given by 
$\mathrm{X}=\left(\mathrm{x}_{1}, \ldots, \mathrm{x}_{\mathrm{p}+1}\right), \sum_{t=1}^{p+1} x_{i}=1$, the wealth accumulation process at time $\mathrm{t}$ satisfies the stochastic differential equation

$$
d W=\left[\sum_{i=1}^{p} x_{i}\left(\mu_{i}-r\right)+r-\frac{1}{2} \sum_{i=1}^{p} x_{i}^{2} \delta_{i}^{2}\right] W d t+\sum_{i=1}^{p} x_{i} W \delta_{i} d Z_{i}
$$

where $\mathrm{dZ}_{\mathrm{i}}$ is standard Brownian motion. The time subscript has been dropped from the equation for convenience. The proportions of wealth invested in risky assets $1, \ldots, \mathrm{p}$ are unconstrained since the proportion invested in the risk free asset can always be chosen (borrowing/lending) to satisfy the budget constraint.

The evaluation of the investment decisions for designated time $t_{0}$ is based upon the conditional distribution of the wealth accumulation process from time $t_{0}$ forward, given the history to time $\mathrm{t}_{0}$. The asset price distributions and investment decisions are fixed based on the information at time $t_{0}$. The capital accumulation process starting from current wealth follows geometric Brownian motion. Furthermore, it has been shown (Karatzas and Shreve, 1988) that inf W(t) $>0$ when $\sum_{i=1}^{p} x_{i}\left(\mu_{i}-r\right)+r-\frac{1}{2} \Sigma x_{i}^{2} \delta_{i}^{2}>0$. It is assumed that this condition is satisfied for all feasible investment strategies.

Corresponding to the wealth process there is a log wealth process. If $\mathrm{V}(\mathrm{t})=$ $\ln \mathrm{W}(\mathrm{t}), \mathrm{t}>\mathrm{t}_{0}$, then $\mathrm{V}(\mathrm{t})$ follows arithmetic Brownian motion with 


$$
\mathrm{dV}=\left[\sum_{i=1}^{p} x_{i}\left(\mu_{1}-r\right)+r-\frac{1}{2} \sum_{i=1}^{p} x_{i}^{2} \delta_{i}^{2}\right] d t+\sum_{i=1}^{p} x_{i} \delta_{i} d Z_{i}
$$

The usual approach to evaluating an investment strategy is to consider accumulated capital or the log of accumulated capital. An alternative approach is to consider the time properties, for example the first passage time to particular wealth levels. The stochastic variables defining these outcomes are $W_{t}\left(X ; w_{t_{0}}\right)=$ accumulated capital (wealth) at time $\mathrm{t}$ starting from wealth $w_{t_{o}}$ at time $\mathrm{t}_{0}<\mathrm{t}$, and investing proportions $\mathrm{X}=\left(\mathrm{x}_{1}, \ldots, \mathrm{x}_{\mathrm{p}+1}\right)$ in investment opportunities, and

$T_{w}\left(X ; w_{t_{0}}\right)=$ first passage time to wealth $w$ starting from wealth $w_{t_{0}}$ at time $\mathrm{t}_{0}$, and investing proportions $\mathrm{X}=\left(\mathrm{x}_{1}, \ldots, \mathrm{x}_{\mathrm{p}+1}\right)$ in investment opportunities.

\section{Wealth}

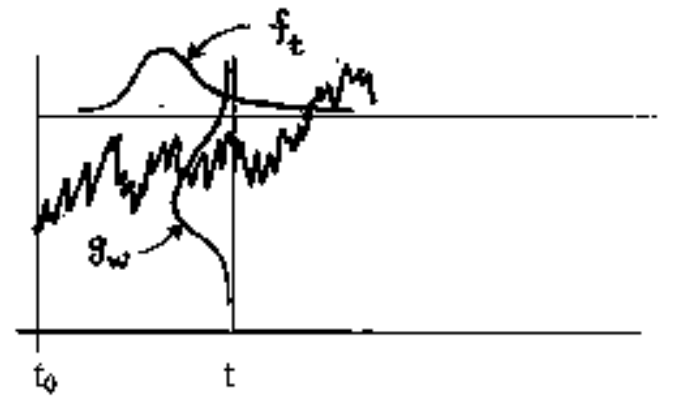

Time

\section{Figure 1: Wealth Trajectory with Distributions}


At time $t_{0}$ the expected rate of return is given by the posterior mean $\mu_{t_{0}}$ The projected wealth and first passage times are derived assuming the fixed value $\mu_{t_{0}}$. In Figure 1 wealth and time distributions are displayed. The functions $f_{t}$ and $g_{w}$ are the conditional densities for $\mathrm{W}_{\mathrm{t}}$ and $\mathrm{T}_{\mathrm{w}}$, respectively. The distribution properties of all trajectories can be described either with $g_{w}$ for all $w>0$ or $f_{t}$ for all $t>t_{0}$. Attention will be focused on $\mathrm{T}_{\mathrm{w}}$ and $\mathrm{g}_{\mathrm{w}}$. Let

$$
\begin{aligned}
& \mu(X)=\sum_{i=1}^{p} x_{i}\left(\mu_{i}-r\right)+r \\
& \sigma^{2}(X)=\sum_{i=1}^{p} x_{i}^{2} \delta_{i}^{2}, \text { and } \\
& D(X)=2 \mu(X)-\sigma^{2}(X) .
\end{aligned}
$$

Then the density $\mathrm{g}_{\mathrm{w}}$ is given by (Cox and Miller, 1970)

$$
g_{w}\left(t \mid X, w_{t_{0}}\right)=\frac{\ln \left(w / w_{t_{0}}\right)}{\sqrt{2 \pi t^{3} \sigma^{2}(X)}} \exp \left[\frac{-\left(\ln \left(w / w_{t_{0}}\right)-\frac{D(X)}{2} t\right)^{2}}{2 t \sigma^{2}(X)}\right] .
$$

The distribution in (15) is the basis for choosing an investment strategy $\mathrm{X}$ at time $\mathrm{t}_{0}$. For example, if $\mathrm{M}$ is a target wealth level then based on the history of asset prices (and posterior mean) an investment decision is taken so that wealth reaches the target in minimal time. This is analogous to setting a course for a destination based on current position. As more price information becomes available (change 
of position) then the posterior mean will be revised, the conditional distribution $\mathrm{g}_{\mathrm{M}}$ will change and a new strategy $\mathrm{X}$ will be adopted to control $\mathrm{g}_{\mathrm{M}}$.

In addition to the distribution for first passage time $T_{w}\left(X ; w_{t_{0}}\right)$, a number of summary measures are relevant to discussion of the performance of a particular investment strategy. Consider wealth levels $m<w_{t_{0}}<M$ and the following measures:

Mean : $G(X)=E\left(T_{M}\left(X ; w_{t_{0}}\right)\right)=\frac{\ln \left(M / w_{t_{0}}\right)}{D(X) / 2}$.

Variance: $V(X)=\sigma^{2}\left(T_{M}\left(X ; w_{t_{0}}\right)\right)=\frac{\ln \left(M / w_{t_{0}}\right) \sigma^{2}(X)}{D^{3}(X) / 4}$.

Probability: $\quad S(X)=\operatorname{Pr}\left[T_{M}\left(x ; w_{t_{0}}\right)<T_{m}\left(x ; w_{t_{0}}\right)\right]$

$$
=\frac{1-\left(m / w_{t_{0}}\right)^{D(X) / \sigma^{2}(X)}}{1-(m / M)^{D(X) / \sigma^{2}(X)}} .
$$

Although these measures are not sufficient to characterize the distribution for $T_{M}\left(X \mid w_{t_{0}}\right)$, they provide a realistic basis for assessing investment decisions. The probability of reaching a target wealth level $M$ before dropping to subsistence level $m$ addresses risk aversion. 


\section{4 - EFFICIENT STRATEGIES}

At point $\mathrm{t}_{0}$ in time, with accumulated capital and the history of asset prices, the investor allocates capital to investment opportunities so that performance is best by accepted criteria. The criteria used here are based upon the first passage time to specified wealth goals. If $F_{w}\left(t \mid X, w_{t_{0}}\right)$ is the distribution function corresponding to the density in (15), then with $w>w_{t_{0}}, T_{w}\left(X_{1} ; w_{t_{0}}\right)$ stochastically dominates $T_{w}\left(X_{2} ; w_{t_{0}}\right)$ iff $\mathrm{F}_{w}\left(t \mid X_{1} ; w_{t_{0}}\right) \geq F_{w}\left(t \mid X_{2} ; w_{t_{0}}\right)$ for every $\mathrm{t}$ with strict inequality for some value $t^{*}$. The implication is that a better investment strategy achieves specified capital accumulation levels in less time.

Working with the full distribution is difficult, so consider the relaxed concept of growth-security dominance: $T_{w}\left(X_{1} ; w_{t_{0}}\right)$ growth-security dominates $T_{w}\left(X_{2} ; w_{t_{0}}\right)$ iff $\left(-G\left(X_{1}\right), S\left(X_{1}\right)\right)>\left(-G\left(X_{2}\right), S\left(X_{2}\right)\right)$. (The notation $\left(\mathrm{a}_{1}, \mathrm{a}_{2}\right)>\left(\mathrm{b}_{1}, \mathrm{~b}_{2}\right)$ implies $a_{i} \geq b_{i}$ with strict inequality in at least one component.) If variance $V(X)$ is used instead of probability $\mathrm{S}(\mathrm{X})$ then $T_{w}\left(X_{1} ; w_{t_{0}}\right)$ mean-variance dominates $T_{w}\left(X_{2} ; w_{t_{0}}\right)$ iff $\left(G\left(X_{1}\right), V\left(X_{1}\right)\right)<\left(G\left(X_{2}\right), V\left(X_{2}\right)\right)$.

It is straightforward from (17) and (18) to write $S(X)=h(V(X))$ and show that $\mathrm{h}$ is monotone decreasing. So growth-security dominance and mean-variance dominance are equivalent. The preference here is for the security measure based on the probability of reaching a wealth goal before falling to subsistence. 
An efficient strategy is one whose first passage time distribution is undominated. So efficient strategies by the growth-security criteria are found from the problem.

$$
\operatorname{Min}_{x}\{G(X) \mid S(X) \geq \gamma, X \text { feasible }\}
$$

(Feasibility requires $\mathrm{D}(\mathrm{X}) \geq 0$.)

Since the growth and security measures have closed forms it is possible to present the format of the growth-security efficient strategy for specific levels of security.

Theorem 1: Consider an investor faced with $m$ risky assets and a risk free asset, where price movements are defined by (7). Based on the history of asset prices to time $t_{0}$ the conditional mean rate of return vector is $\mu_{t_{0}}$ and the covariance matrix is $\Delta_{t_{0}}$. With wealth goal $M$, subsistence level $m$, security specification $\gamma$, and current wealth $w_{t_{0}}$, the growth-security efficient strategy for risky assets $X=\left(x_{1}\right.$, ..., $x_{p}$ ) has the form

$$
X=p\left(w_{t_{0}}, \gamma, m, M\right) \Delta_{t_{0}}^{-1}\left(\mu_{t_{0}}-r e\right)
$$

Proof:

Consider the growth-security efficiency problem $\min \{\mathrm{G}(\mathrm{X}) \mid \mathrm{S}(\mathrm{X}) \geq \gamma, \mathrm{X}$ feasible $\}$. Let $m={ }_{t_{0}} / k$ and $M=k^{\beta} w_{t_{0}}$, where $\mathrm{k}>1$ and $\beta>0$. Then the efficiency problem is 


$$
\operatorname{Max}\left\{D(X) \mid\left[\frac{1-k^{-E(X)}}{1-k^{-(\beta+1) E(X)}}\right] \geq \gamma, X \text { feasible }\right\},
$$

where $D(X)=2 \mu(X)-\sigma^{2}(X)$, and $E(X)=D(X) / \sigma^{2}(X)$.

Letting $\mathrm{y}=\mathrm{k}^{-\mathrm{E}(\mathrm{X})}$, the constraint can be written as $\gamma \mathrm{y}^{\beta+1}-\mathrm{y}+(1-\gamma) \geq 0$.

Consider the minimum positive root $\mathrm{y}^{*}$ of the equation $\gamma \mathrm{y}^{\beta+1}-\mathrm{y}+(1-\gamma)=0$, where $\mathrm{y}^{*} \leq 1$ since $\mathrm{y}^{*}=1$ is a root. Then $\gamma \mathrm{y}^{\beta+1}-\mathrm{y}+(1-\gamma) \geq 0$ for $\mathrm{y} \leq \mathrm{y}^{*}$ and the constraint is satisfied iff $\mathrm{k}^{-\mathrm{E}(\mathrm{X})} \leq \mathrm{y}^{*}$ or $\mathrm{E}(\mathrm{X}) \geq-\left(\log \mathrm{y}^{*} / \log \mathrm{k}\right)=\mathrm{q}^{*} \geq 0$, where $\mathrm{q}^{*}$ depends upon the specifications $m, M$ and $\gamma$. From the definition of $E(X), E(X) \geq$ $q^{*}$ iff $2 \mu(X)-\left(1+q^{*}\right) \sigma^{2}(X) \geq 0$.

Therefore the growth-security efficiency problem has the form

$$
\underset{X}{\operatorname{Max}}\left\{2 \mu(X)-\sigma^{2}(X) \mid 2 \mu(X)-(1+q *) \sigma^{2}(X) \geq 0, X \text { feasible }\right\} \text { for } q^{*}=-\left(\log y^{*} / \log k\right) .
$$

If $\mathrm{X}^{*}$ is a solution to this problem then it also solves, for appropriate multiplier $\mathrm{p}^{*}$, the Lagrangian problem: $\max _{x} \Psi\left(X, p^{*}\right)$,

where $\Psi\left(X, p^{*}\right)=2 \mu(X)-\sigma^{2}(X)+p^{*}\left(2 \mu(X)-\left(1+q^{*}\right) \sigma^{2}(X)\right)$. The first order conditions $\nabla_{X} \Psi\left(X^{*}, p^{*}\right)=0$ imply that the optimal $X^{*}$ satisfies the linear system $2\left(1+p^{*}\right)\left(\mu_{i}-r\right)-2\left(1+p^{*}\left(1+q^{*}\right)\right)\left[x_{i} \delta_{i}^{2}\right]=0, i=1, \ldots, p$.

The solution to this system is

$$
X^{*}=p\left(w_{t_{0}}, \gamma, m, M\right) \Delta_{t_{0}}^{-1}\left(\mu_{t_{0}}-r e\right)
$$


where $p\left(w_{t_{0}}, \gamma, m, M\right)=\frac{1+p^{*}}{1+p^{*}\left(1+q^{*}\right)}$.

The growth-security efficient strategies have a very simple structure.

Consider a weak security requirement where $\gamma \leq[1 /(\beta+1)]$. In this case $\gamma^{\beta+1}-y+(1-\gamma)=0$ has as a minimum positive root $\mathrm{y}^{*}=1$ and therefore $\mathrm{q}^{*}=-\log \mathrm{y}^{*} / \log \mathrm{k}=0$, giving $p\left(w_{t_{0}}, \gamma, m, M\right)=1$. The investment in risky assets is $X^{*}=\Delta_{t_{0}}^{-1}\left(\mu_{t_{0}}-r e\right)$, the optimal growth (Kelly) strategy based upon the conditional mean and covariance from the history of asset prices. This can be called a Bayesian Kelly strategy, see Browne and Whitt, (1996).

When the security requirement is stronger $(\gamma>[1 / \beta+1])$ then $\mathrm{y}^{*}<1, \mathrm{q}^{*}>0$ and $p\left(w_{t_{0}}, \gamma, m, M\right)<1$. In this case the optimal investment in risky assets is a fraction of the optimal growth strategy, or a fractional Bayesian Kelly strategy. As the fraction in risky assets decreases, the fraction of wealth allocated to the risk free asset increases proportionately.

\section{5 - SECURITY AND WEALTH EFFECTS}

Having developed the form of growth-security efficient strategies, the impact of various inputs on the fraction $p\left(w_{t_{0}}, \gamma, m, M\right)$ can be investigated. The 
specific inputs considered are (i) security level, (ii) wealth goals, and (iii) current wealth.

(iii) Theorem 2. Consider the growth-security efficient investor with current wealth $w_{t_{0}}$, subsistence level $m=k^{-1} w_{t_{0}}$, goal $M=k^{\beta} w_{t_{0}}$ and security specification $\gamma$. (i) The fraction of wealth allocated to the risky assets is a decreasing function of the security level $\gamma$ for given wealth levels $m$ and $M$, and current wealth $w_{t_{0}}$. (ii) The fraction of wealth allocated to risky assets is a nonincreasing function of the subsistence level m, other input values held constant. (iii) The fraction of wealth allocated to risky assets is a nondecreasing function of $M$, other input values held constant. (iv) The fraction of wealth allocated to risky assets is a nondecreasing function of current wealth $w_{t_{0}}$, other input values held constant.

Proof: With the form of the optimal solution in (20) and $\Delta_{t_{0}}^{-1}\left(\mu_{t_{0}}-r e\right)$ fixed, the security condition can be written as

$$
S(p)=\frac{1-k^{-E(p)}}{1-k^{-(\beta+1) E(p)}} .
$$


with $E(p)=D(p) / \sigma^{2}(p)=\frac{A}{p}+\frac{B}{p^{2}}-1$ for appropriate constants A and B. So

$\frac{\partial}{\partial p} E(p)<0$. Also $\frac{\partial S}{\partial E}>0$ and therefore $\frac{\partial S}{\partial p}<0$. That is, the security level is a decreasing function of the fraction of wealth allocated to the risky assets, and the optimal fraction satisfies $p=S^{-1}(\gamma)$. Then $\frac{\partial p}{\partial \gamma}=\left(\frac{\partial S}{\partial p}\right)^{-1}<0$, establishing (i).

The other parts of the proposition follow in the same way using simple calculus.

\section{6-FINAL REMARKS}

This paper considers a multi-asset investment problem where the distribution of asset prices has random parameters. The criteria for assessing investment strategies is the first passage time to specified wealth goals. The optimal (efficient) strategy is based on the growth optimal (Kelly) strategy. In particular, the investor optimally blends the Kelly portfolio with cash (fractional Kelly) to obtain a required level of security.

The fractional Kelly strategy is also optimal from a wealth perspective. That is, if the investor's objective is to maximize the median accumulated capital (wealth) to the time horizon $\mathrm{t}$, subject to the probability $\gamma$ that wealth at time $\mathrm{t}$ exceeds a wealth goal, then the optimal strategy is fractional Kelly. This result depends upon the assumption of lognormality of asset returns. 
The Kelly strategy and the fractional Kelly strategy at a point in time are determined by the posterior mean of the random rate of return on assets, given the history of asset prices to date. As future prices are realized the posterior means are revised generating a new Bayes investment strategy.

In practice the parameters in the Bayes model for asset prices may be estimated using empirical Bayes methods. Some common empirical Bayes estimators have been displayed. 


\section{7 - REFERENCES}

Algoet, P.H., and T.M. Dover (1988). Asymptotic optimality and asymptotic equipartition properties of log-optimum investment. Annals of Probability, $16.2,876-898$.

Breiman, L. (1960). Investment policies for expanding business optimal in a longrun sense. Naval Research Logistics Quarterly, 7.4, 647-651.

Breiman, L. (1961). Optimal gambling systems for favorable games, in Proceedings of the $4^{\text {th }}$ Berkeley Symposium on Mathematics, Statistics and Probability, 1, 63-68.

Brennan, M.J. (1998). The Role of Learning in Dynamic Portfolio Decisions. Working paper, University of California, Los Angelos.

Browne, S. and Whitt, W. (1996). Portfolio Choice and the Bayesian Kelly Criterion. Adv. Appl. Prob., 28, 1145-1176.

Burkhardt, T. (1998). A Mean-Variance of First Passage Time Approach to Portfolio Selection in a Lognormal World. Presentation at VIII International Conference on Stochastic Programming, Vancouver, August.

Clark, R., and Ziemba, W.T. (1987). Playing the turn-of-the-year effect with index futures. Operations Research, 35, 799-813.

Cox, D.R. and H.D. Miller (1970). The Theory of Stochastic Processes. Methuen \& Co. Ltd., London. 
Efron, B. and Morris, C. (1972). Empirical Bayes on Vector Observations - An Extension of Stein's Method. Biometrica, 59, 335-347.

Grauer, R.R., and Hakansson, N.H. (1986). A half century of returns on levered and unlevered portfolios of stocks, bonds and bills, with and without small stocks. Journal of Business, 59.2, 287-318.

Grauer, R.R., and Hakansson, N.H. (1987). Gains from international diversification: 1968-85 returns on portfolios of stocks and bonds. Journal of Finance, 42, 721-739.

Hakansson, N.H. (1970). Optimal investment and consumption strategies under risk for a class of utility functions. Econometrica, 38, 587-607.

Hakansson, N.H. (1971). Capital growth and mean-variance approach to portfolio selection. Journal of Financial and Quantitative Analysis, 6, 517-557.

Hausch, D.B., and Ziemba, W.T. (1985). Transactions costs, extent of inefficiencies, entries and multiple wagers in a racetrack betting model. Management Science, 31.4, 381-392.

Hausch, D.B., Ziemba, W.T., and Rubinstein, M. (1981). Efficiency of the market for racetrack betting. Management Science, 27, 1435-1452.

Karatzas, I. And Shreve, S. (1988). Brownian Motion and Stochastic Calculus, Springer-Verlag, New York. 
Kelly, J. (1956). A new interpretation of information rate. Bell System Technology Journal, 35, 917-926.

MacLean, L.C. and Weldon, K.L. (1996). Estimating Multivariate Random Effects without Replication. Commun. Statist. Theory Meth., 24(7), 1447-1469.

MacLean, L.C., Ziemba, W.T., and Blazenko, G. (1992). Growth versus security in dynamic investment analysis. Management Science, 38, 1562-1585.

Markowitz, H.M. (1952). Portfolio Selection. Journal of Finance, 7, 77-91.

Markowitz, H.M. (1976). Investment for the long run: New evidence for an old rule. Journal of Finance, 31.5, 1273-1286.

Markowitz, H.M. (1987). Mean-Variance Analysis in Portfolio Choice and Capital Markets. Basil Blackwell, New York.

Mulvey, J.M., and Vladimirou, H. (1992). Stochastic network programming for financial planning problems. Management Science, 38, 1642-1664.

Thorp, E.O. (1975). Portfolio choice and the Kelly criterion. In: Stochastic Optimization Models in Finance (eds. W.T. Ziemba and R.G. Vickson). Academic Press, New York, 599-619. 